\title{
Improved Image Denoising Based on 3D Collaborative Filtering
}

\author{
Xuemei Wang ${ }^{1}$, Dengyin Zhang ${ }^{2}$, Min Zhu ${ }^{3}$, Yingtian $\mathrm{Ji}^{2}$ and Jin Wang ${ }^{4}$ \\ ${ }^{l}$ College of Computer, Nanjing University of Posts and Telecommunications, \\ Nanjing, 210003, China \\ ${ }^{2}$ Internet of Things Technology Park, Nanjing University of Posts and \\ Telecommunications, Nanjing, 210003, China \\ ${ }^{3}$ Information \& Technical Department, Wenzhou Vocational \& Technical College, \\ wenzhou 325000 \\ ${ }^{4}$ College of Information Engineering, Yangzhou University, Yangzhou 225009,
}

China

\begin{abstract}
As the state-of-art denoising method, BM3D is capable of achieving good denoising performance by exploiting both the non-local characteristics and sparsity prior knowledge of images. Nevertheless, experimental results show that the dissimilarity measurement defined in BM3D sometimes results in grouping patches with distinct structure. Inspired by the fact about the different impact of noise on patches with various structures, we propose a structure-adaptive image denoising method with $3 D$ collaborative filtering by optimizing the block matching procedure. In our method, the similarity in the variance between patches is incorporated in block matching procedure. Besides, based on the prior knowledge of correlation among patches in the same neighborhood, the spatial distance between the reference patch and the candidate is also taken into account when measuring patches' dissimilarity. Several numerical experiments demonstrate that the proposed approach achieve better results in PSNR and visual effect than original BM3D.
\end{abstract}

Keywords: block matching, spatial similarity, variance, structure-adaptive

\section{Introduction}

Image denoising problem has drawn considerable research attention in past decades. It aims at separating the true signal from random noise. All the existing approaches rely on some explicit or implicit assumptions or prior knowledge of the noise-free image. Traditional means, such as Gaussian spatial filtering, exploit spatial similarity to remove noise. The non-local mean (NLM) [1] denoising method is a typical up-to-date example of this type. It exploits the inter-patch correlations and adopts neighborhood filters to reduce the noise by averaging similar pixels. However, due to the averaging operation, NLM method may give rise to over-smooth visual effect. In the recent years, sparse representation based methods have proven to be more effective in denoising. They take full advantage of sparsity prior knowledge of image in transform domain to attenuate noise. A typical instance is Wavelet shrinkage approach, which performs thresholding operation on wavelet coefficients to yield approximate estimate of original true image. To achieve good sparsity for spatially localized details, a variety of multiresolution transforms or overcomplete representations have been developed in shrinkage denoising methods [2-4].

BM3D [5] integrates both sparse representation and non-local averaging operation, and is widely recognized as the state-of-art denoising technique. By grouping similar patches together to form 3D array, it can achieve an enhanced sparse representation in transform domain, which ensures its outstanding denoising performance. 


\section{Related Work about BM3D}

There are two critical procedures in BM3D: block matching and 3D collaborative filtering. Block matching is performed to find patches similar to a given reference one, and in the original $\mathrm{BM} 3 \mathrm{D}$, the 2-norm distance is adopted to measure patches' dissimilarity. To prevent the output-PSNR from sharp drop in case of heavy noise, BM3D introduces coarse prefiltering before the block-distance measurement, by applying hardthresholding operator on the coefficients of a normalized 2D linear transform. Experimental results show that prefiltering may bring visual blocking effect and result in the removal of the true image signal [6]. To achieve better visual effect, Omid [7] proposed to remove prefiltering and adjust the parameters adaptively according to the estimated noise level.

In two denoising stages, collaborative filtering is implemented through hard thresholding and wiener filtering respectively. In collaborative filtering, it is likely for noise and true signal to be inseparable if patches can not be sparsely represented in 3D transform domain. In this case, it happens inevitably that filtering operation may remove partial energy of image signal. Furthermore, edges in images may be blurred since the weighted averaging behavior in aggregation actually acts as a low-pass filter. To mitigate this drawback, Chen [8] presented a bounded BM3D scheme, in which image is partitioned into multiple regions and a partial block matching is conducted when the reference patch contains several segments separated by edge. In fact, it is difficult to recognize the boundary of multiple regions in heavy noise, and erroneous region partitioning will pose a negative effect in block matching. In addition, Dabov [9] proposes a shape-adaptive collaborative filtering method, in which the shape of neighborhood can be selected adaptively and Shape-Adaptive DCT takes the place of DCT to perform 2D transform. On the basis of [9], Dabov [10] also incorporates Principle Component Analysis (PCA) into BM3D and designs the improved BM3D method with shapeadaptive PCA. Both methods in $[9,10]$ are invalid for images in heavy noise, because it is tough to discern the shape of neighborhood when images are immerged in strong noise.

In this paper, we examine the dissimilarity measurement method in the original BM3D through the experimental results of block matching, and propose an improvement on BM3D. In our method, structure-adaptive block matching procedure is adopted, and different types of patches are grouped in different ways. The similarity in the variance is incorporated in block matching procedure. Besides, based on the prior knowledge of correlation among patches in the same neighborhood, the spatial distance between the reference patch and the candidate is also taken into account when measuring patches' dissimilarity. Numerical experimental results illustrate that the proposed method could achieve better performance in image denoising.

This paper is organized as follows. In Section 3, an improved BM3D denoising scheme is proposed. The experimental results are presented in Section 4. Section 5 concludes the paper.

\section{Structure-adaptive BM3D Denoising Scheme (SA-BM3D)}

In $\mathrm{BM} 3 \mathrm{D}$, grouping procedure collects similar patches into 3D array through block matching. Only patches whose dissimilarity with respect to the reference one is smaller than a fixed threshold are considered similar. The original BM3D defines to measure the dissimilarity between the reference fragment $Z_{x_{R}}$ and the candidate $Z_{x}$.

$$
d\left(Z_{x_{R}}, Z_{x}\right)=\frac{\left\|Z_{x_{R}}-Z_{x}\right\|_{2}^{2}}{\left(N_{1}^{h t}\right)^{2}}
$$

Generally, it is effective to compute the dissimilarity using the 2-norm distance illustrated in Eq. (1). Nevertheless, experimental results show that Eq. (1) will result in 
erroneous grouping possibly even for clean images. For example, as shown in Figure 1(a), if the procedure of block matching proceeds with Eq. (1) in a non-overlapping manner, the eight rectangles highlighted will be regarded as similar ones. Obviously, the block on the left-down differs greatly from the other seven ones in texture, and it is regarded as 'pseudo-similar' and should be weed out. To eliminate the erroneous grouping like this, some extra information about blocks' structure needs to be merged into dissimilarity measurement. Recall that variance is capable of measuring the complexity of image patches in texture or structure to some extent, so patches with similar structure may have similar variances. It occurs to us that better grouping result may be achieved by excluding those patches with small dissimilarity measured by Eq. (1) and large difference in variance from the reference one. Figure 1(b) shows the corresponding matching result when variance is integrated into grouping procedure as above. Obviously, the pseudosimilar block in Figure 1 (a) is eliminated and no longer regarded as similar one.

Actually, block matching procedure is performed on noisy images. Once image patches are contaminated with random Gaussian noise, they experience an unexpected change in pixel intensity. And erroneous grouping is often the case if only the 2-norm distance in Eq. (1) is adopted in dissimilarity measurement. Then does variance work in grouping for noisy images as well? In order to evaluate whether the integration of variance into block matching make sense, it is necessary to examine the impact of noise on patches' variance. In Figure 2(a), we select four patches with distinct structures (denoted by '1\#' '2\#', '3\#' and '4\#') from lena image, and the trend these patches' variances change with noise level is illustrated in Figure 2 (c). As seen from Figure 2(c), when patches are polluted with noise, the variance of them will increase inevitably. And stronger noise results in greater variance. In fact, what we are really concerned about is the relative change rate in variance among distinct patches. To achieve this goal, we propose the approach to normalize the patch variance, as shown in Eq. (2) and (3). In Eq. (2), $V$ denotes the set of patches' variance and $V^{\prime}$ is the deviation of $V$ from the average. Eq. (3) maps the result from Eq.(2) to the corresponding normalized value in the range between 0 and 1.

$$
\begin{aligned}
& V^{\prime}=V-\operatorname{mean}(V) \\
& \text { normalize }(V)=\left(V^{\prime}+\left|\min \left(V^{\prime}\right)\right|\right) /\left(\max \left(V^{\prime}\right)-\min \left(V^{\prime}\right)\right)
\end{aligned}
$$

Figure 3 shows the normalized variance defined in Eq. (3) of 130 patches extracted from two standard gray images, 'lena' and 'barbara', in different noise levels. As seen from Figure 3, if the image is polluted by a small amount of noise, the normalized variance of noisy patches nearly coincides with that of the corresponding clean ones. When noise intensity increases, much burr appears in the regions of relatively small variance. For patches with smooth structure or simple regular texture, most of energy concentrate in a narrow range of gray level, as shown in Figure 2.(b). And their variances are relatively small compared to patches with complex texture. Hence, we can infer that noise imposes less impact on variance change of patches with more detail information, and there is a significant uncertainty in variance change rate for smooth patches or those with simple regular texture. In other words, for two noisy patches with smooth structure, they are more likely to differ greatly in variance, even if they resemble each other in the absence of noise. Therefore, it is more appropriate for noisy patches with complex structure to take variance into account when grouping. 


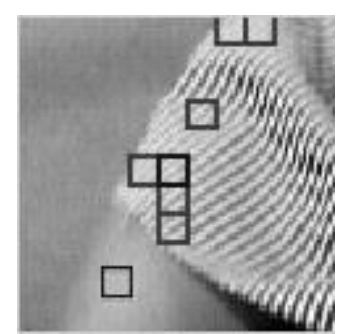

(a)

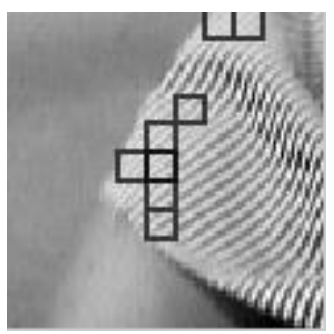

(b)

Figure 1. Comparison of Grouping Results

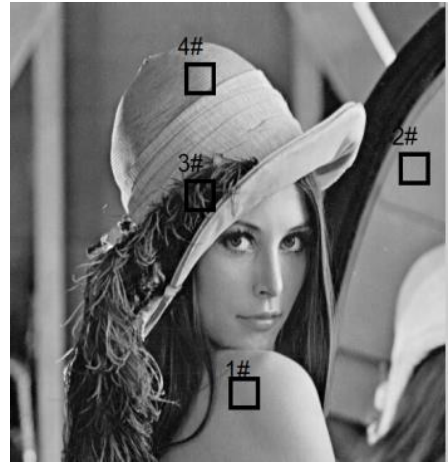

(a)
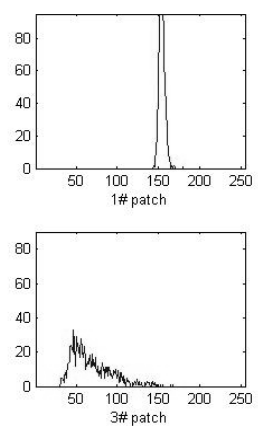

$100 \quad 150$
$3 \#$ patch
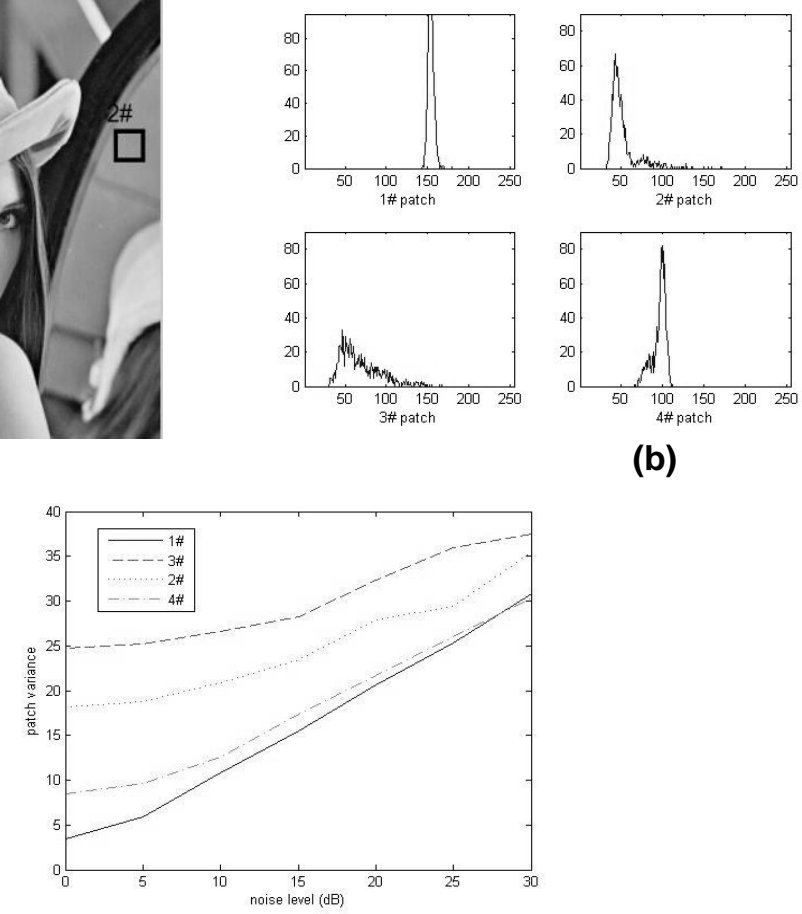

(b)

(c)

Figure 2. (a) Four Patches Denoted by '1\#','2\#','3\#' and '4\#' in Lena Image (b) Histogram of these Four Patches (c) Variance Change of Four Patches with Noise Level
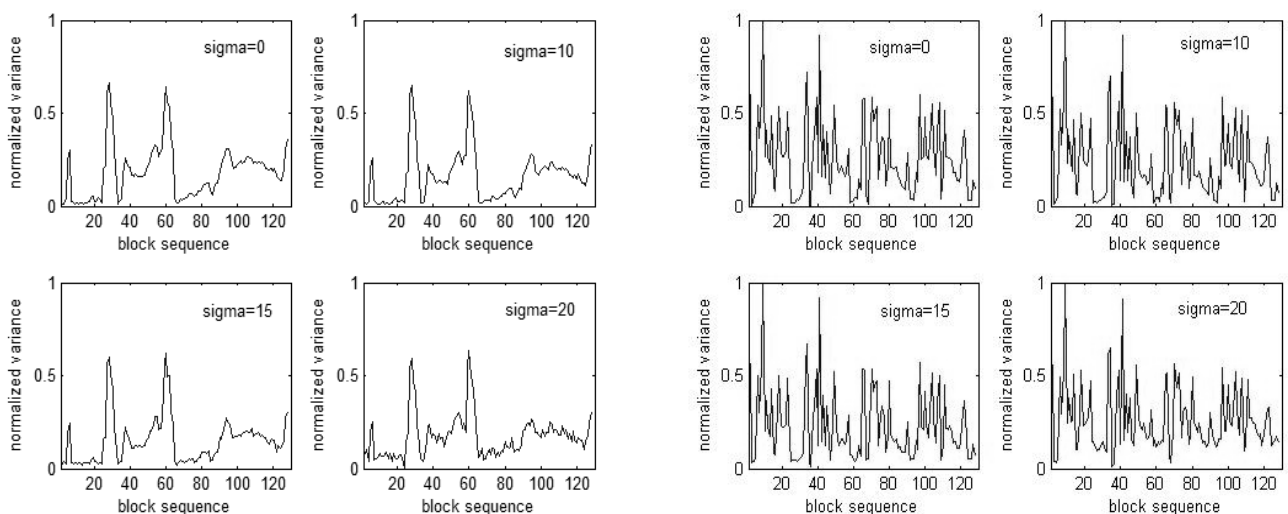

(a) lena
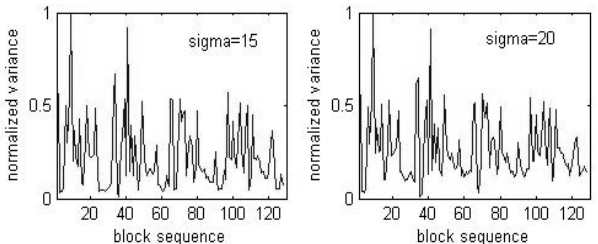

(b) barbara

Figure 3. Normalized Variance of Block Sequence from Lena and Barbara in Different Noise Levels 


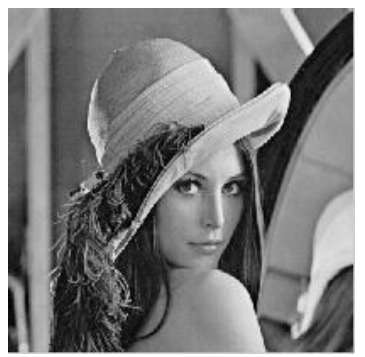

(a) Lena Image

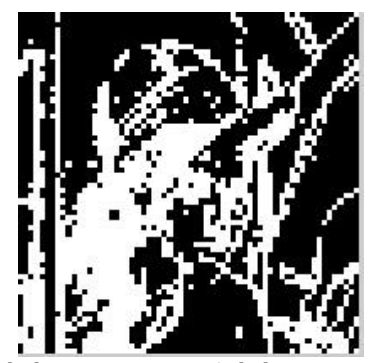

(b) Results of (a)

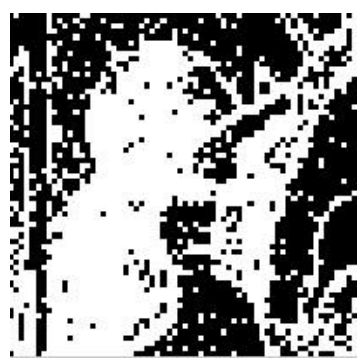

(c) Results of Noisy Lena $(\sigma=5)$

Figure 4. Classification Results from DCT Method: the Black and White Indicate Smooth and Non-smooth Patches Respectively

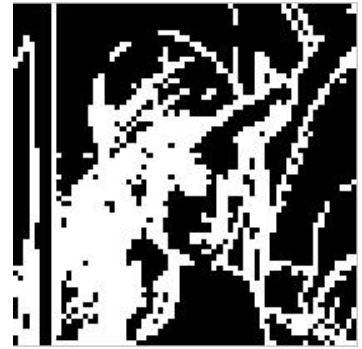

(a) clean image

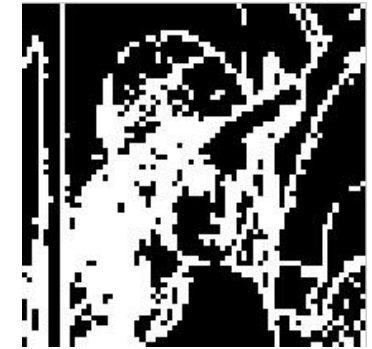

(b) noisy image $(\sigma=10)$

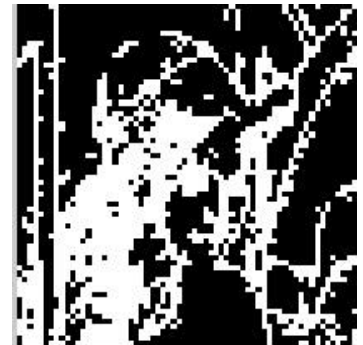

(c) noisy image $(\sigma=20)$

Figure 5. Classification Results from Variance based Method: the Black and White Indicate Smooth and Non-smooth Patches Respectively

In the original $\mathrm{BM} 3 \mathrm{D}$, when the clean image encounters strong noise, its matching results differ greatly from that of the corresponding noise-free one, and it is more likely to produce 3D group comprising of patches scattered in a wide range. Especially, since smooth patches are more susceptible to noise than non-smooth ones, the block matching procedure on smooth patches is prone to generate more 'pseudo-similar' patches. Due to the decrease in inter-patch correlation resulting from the presence of pseudo-similar blocks, the denoising performance in collaborative filtering suffers.

As a matter of fact, in the smooth region of a given image, patches in the neighborhood are more likely to be correlative and become mutual similar ones. This fact inspires us to take the local spatial distance between patches into account when computing the dissimilarity. That is, for smooth region, nearby patches take precedence to be selected as similar ones. So we need to introduce an additional scalar weight to the original 2-norm definition of dissimilarity, the value of which depends on the spatial distance between the candidate patch and the reference one. Intuitively, small weight should be assigned to patches close to the reference one. Therefore, we modify Eq. (1) into

$d^{p}\left(Z_{x_{R}}, Z_{x}\right)=w\left(Z_{x_{R}}, Z_{x}\right) \frac{\left\|Z_{x_{R}}-Z_{x}\right\|_{2}^{2}}{\left(N_{1}^{h t}\right)^{2}}$

Where $w\left(Z_{x_{R}}, Z_{x}\right)$ the non-negative is valued weight and defined in Eq. (5). Here, our choice for the weight is the sigmoid function of the spatial distance between $Z_{x_{R}}$ and $Z_{x}$. If $\left\|x_{R}-x\right\|_{2}$ is large, then $w\left(Z_{x_{R}}, Z_{x}\right)$ is close to 1 . The parameter ${ }^{h}$ controls how quickly patch's weight increases with its distance from the reference one.

$w\left(Z_{x_{R}}, Z_{x}\right)=\frac{1}{1+e^{\left(-\left\|x_{R}-x\right\|_{2} / h\right)}}$
$G_{x_{R}}^{h t}=\left\{x \in X: d^{p}\left(Z_{x_{R}}, Z_{x}\right) \leq \tau_{\text {match }}^{h t}\right\}$ 
$G_{x_{R}}^{h t}=\left\{x \in X: d\left(Z_{x_{R}}, Z_{x}\right) \leq \tau_{1}^{h t} \& \& \operatorname{abs}\left(\operatorname{std}\left(Z_{x_{R}}\right)-\operatorname{std}\left(Z_{x}\right)\right) \leq \tau_{2}^{h t}\right\}$

On the basis of the above analysis, we put forward a structure-adaptive grouping approach. The basic idea is to determine block matching scheme adaptively according to the structure type of the reference patch. For the sake of simplicity, we classify all blocks into two types, smooth and non-smooth. Smooth patches are homogeneous while nonsmooth ones contain rich texture details. For a smooth reference patch, Eq. (6) is used to execute the pair-wise testing of the inter-patch similarity. The patches whose distance from the reference one is smaller than the given threshold are considered similar and are subsequently grouped. On the other hand, if the reference block is anisotropy and contains complex texture details, block matching procedure adopts dual thresholding operator to select similar patches, as shown in Eq. (7). That is, besides the similarity testing in form of 2-norm distance, an extra judgment about the variance similarity is also required simultaneously. In other words, the patches are considered as similar ones only when dissimilarity in both pixel and variance do not exceed ${ }^{\tau_{1}^{h t}}$ and ${ }^{\tau_{2}^{h t}}$ at the same time. Due to the wide variations in variance of different images, it is inappropriate to configure ${ }^{\tau^{h t}}$ as a fixed threshold. In our proposed method, ${ }^{\tau_{2}^{h t}}$ is configured dynamically as the median of the absolute variance differences of all candidates from the reference patch.

Since two types of patches vary in grouping procedure, it becomes a key issue to recognize the type of a given patch. A variety of methods are available to implement it. But most of them are invalid in correctly classifying polluted blocks. For instance, Zhang [11] exploited the prior knowledge of DCT energy distribution to perform classification. For a noise-free image, it works well and is capable of achieve good results. Nevertheless, the energy distribution of patches will be destructed by the presence of noise because most of the noise energy concentrate on the medium or high frequency band in DCT transform domain. It can be seen from Figure4 that even with a small amount of noise injected, numerous misclassifications arise. In addition, average gradient or information entropy can also be adopted in patch type recognition, and they are prone to misclassification for noisy images as well.

As mentioned above, there is great difference in the variance between smooth and nonsmooth patches, and non-smooth patches usually possess larger variance than smooth ones in general. And this prior knowledge of variance could be applied to roughly classify image patches in a simple way. That is, patches with variance larger than average are recognized as non-smooth, otherwise, as smooth. It proves to be effective to perform classification using variance despite of its simplicity. As shown in Figure5, the classification results are satisfactory even for noisy images.

\section{Simulation Results}

To validate the improvement of the proposed approach over original BM3D, we implement the corresponding simulation programs in matlabR2012a. The standard test images we select include Barbara, Lena, peppers, which show different features in texture structure. And the configuration of parameters adopted in our simulation refers to the normal profile in [5]. Table 1 compares the PSNR (dB) performance of the proposed algorithm with BM3D in denoising images with noise level at $\sigma=20,30,40,50,60$ respectively. In Table 1, the column of 'scheme I' shows the PSNR results when Eq. (6) is adopted to group similar patches; The column of 'scheme II' displays the PSNR results when dual-thresholding in Eq. (7) is applied to all reference patches to select similar ones. The right-most column provides the denoising results when the grouping procedure uses the structure-adaptive block matching method proposed in Section 3, which combines scheme I and scheme II. Obviously, compared with original BM3D, all three schemes identified with 'scheme I', 'scheme II' and 'combined' achieve better results in PSNR. 
Moreover, it can be clearly seen from Table I that scheme II outperforms scheme I in general cases while 'peppers' is an exception. When denoising 'peppers' image with noise level at $\sigma \geq 40$ scheme I gets better outcome than scheme II. The major reason for this exception is that in 'peppers', the smooth patches account for most part and consequently, the effect of denoising assisted by variance in heavy noise is weakened. Also we note that the joint scheme identified with 'combined' is inferior to scheme II when denoising the 'barbara' image, which may be caused by existence of lots of texture in the image.

Figure 5 compares the visual effect of denoising images in the proposed method and original BM3D. From Figure 5, we can see that the proposed method can retain more detail information and images in column (d) contains more clear texture than that of column (c).

\section{Conclusion}

In order to group as few pseudo-similar patches as possible, the structure-adaptive block matching algorithm is presented in this paper. Inspired by the experimental results illustrating the impact of noise on patches with various structures, we propose an improvement on the original 2-norm distance measurement by incorporating the similarity in the variance between patches to find mutual similar ones. Besides, based on the prior knowledge of correlation among patches in the same neighborhood, the 2-norm dissimilarity measurement is modified by adding an extra scalar weight, which depends on the spatial distance between the reference patch and the candidate. Simulation results demonstrate the proposed method outperforms the original BM3D in both PSNR and visual quality at the cost of extra computational overhead.

Table 1. PSNR(dB) COMPARISON BETWEEN our Proposed Method AND Original BM3D

\begin{tabular}{|c|c|c|c|c|c|}
\hline$\sigma$ & image & BM3D & Scheme I & Scheme II & Combined \\
\hline \multirow{3}{*}{20} & Lena & 32.75 & 32.78 & 32.86 & 32.87 \\
\cline { 2 - 6 } & Peppers & 32.37 & 32.41 & 32.44 & 32.45 \\
\cline { 2 - 6 } & Barbara & 31.90 & 31.96 & 32.09 & 32.06 \\
\hline \multirow{3}{*}{30} & Lena & 30.88 & 30.94 & 30.96 & 30.98 \\
\cline { 2 - 6 } & Peppers & 30.76 & 30.81 & 30.83 & 30.85 \\
\cline { 2 - 6 } & Barbara & 29.65 & 29.72 & 29.79 & 29.77 \\
\hline 40 & Lena & 29.44 & 29.52 & 29.53 & 29.55 \\
\cline { 2 - 6 } & Peppers & 29.30 & 29.33 & 29.31 & 29.39 \\
\cline { 2 - 6 } & Barbara & 27.86 & 27.91 & 27.97 & 27.95 \\
\hline \multirow{3}{*}{50} & Lena & 28.74 & 28.83 & 28.88 & 28.90 \\
\cline { 2 - 6 } & Peppers & 28.75 & 28.82 & 28.79 & 27.83 \\
\cline { 2 - 6 } & Barbara & 27.14 & 27.21 & 27.33 & 28.04 \\
\hline \multirow{2}{*}{60} & Lena & 27.89 & 27.98 & 28.03 & 27.91 \\
\cline { 2 - 6 } & Peppers & 27.78 & 27.87 & 27.83 & 26.43 \\
\cline { 2 - 6 } & Barbara & 26.24 & 26.33 & 26.42 & \\
\hline
\end{tabular}



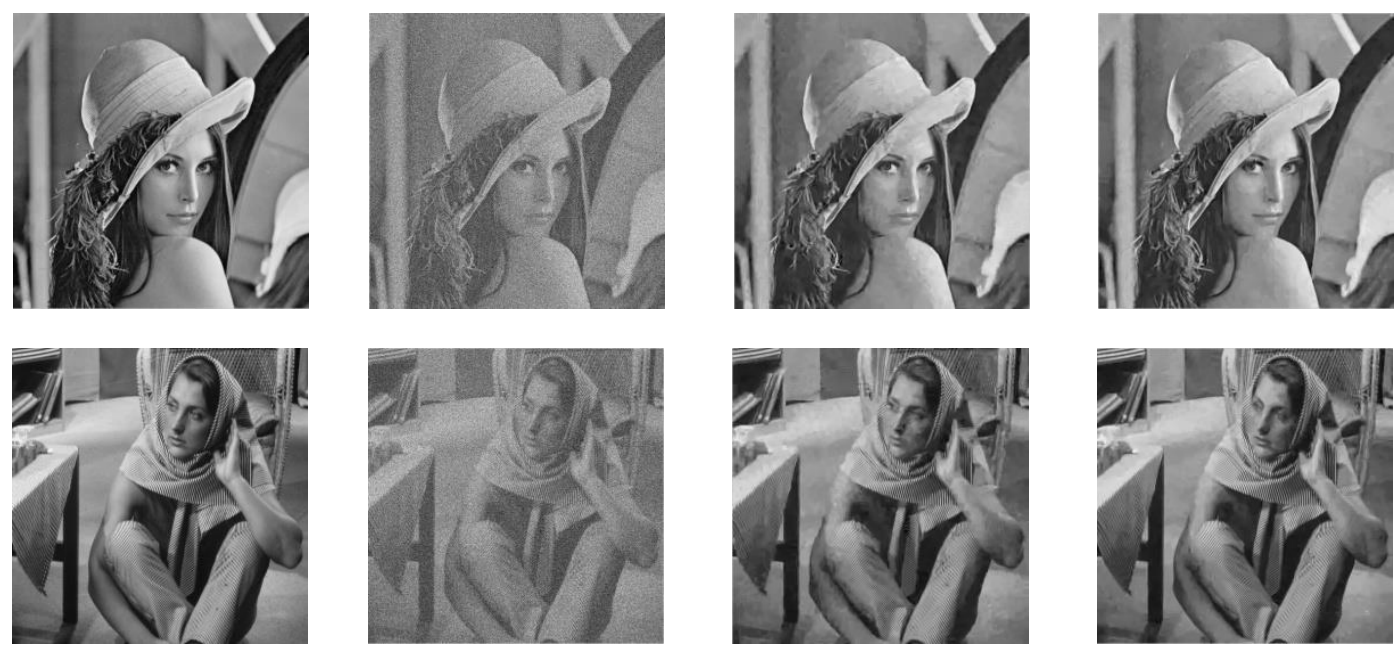

(a) Clean Image (b) Noisy Image $(\sigma=40)$

(c) Original BM3D (d) the Proposed Method

\section{Figure 5. Comparison of Visual Denoising Effect}

\section{ACKNOWLEDGMENTS}

This research work is supported by the National Natural Science Foundations of P.R. China (NSFC) under Grant (61071093), National 863 Program (2010AA701202), SwedenAsian International Cooperation Project (348-2008-6212), Jiangsu Province Major Technology Support Program (BE2012849), Jiangsu Province industry-university-research prospective joint research project (BY2014014), Jiangsu Province Graduate Research Innovation Project No.CXZZ13_0476 and No.KYLX_0812.

\section{References}

[1] A. Buades, B. Coll and J. M. Morel, "On image denoising methods", SIAM: Multi-scale Modeling and Simulation, vol. 4, no. 2, (2005).

[2] J. Portilla, V. Strela, M. Wainwright and E. P. Simoncelli, "Image denoising using a scale mixture of Gaussians in the wavelet domain”, IEEE Trans. Image Process., vol. 12, no. 11, (2003).

[3] M. Elad and M. Aharon, "Image denoising via sparse and redundant representations over learned dictionaries", IEEE Trans. on Image Process, vol. 15, no. 12, (2006).

[4] K. Dabov, A. Foi, V. Katkovnik and K. Egiazarian, "Image denoising by sparse 3D transform domain collaborative filtering", IEEE Trans. Image Process, vol. 16, no. 8, (2007).

[5] Y. K. Hou, C. X. Zhao, D. Y. Yang and Y. Cheng, "Comment on Image denoising by sparse 3D transform-domain collaborative filtering", IEEE Trans. Image Process, vol. 20, no. 1, (2011).

[6] O. Pakdelazar and G. Rezai-Rad, "Improvement of BM3D algorithm and employment to satellite and CFA images denoising", International Journal of Information Sciences and Techniques (IJIST), vol. 1, no. 3, (2011).

[7] Q. Chen and D. Wu, "Image denoising by bounded block matching and 3D filtering", Signal Processing, vol. 90, no. 9, (2010).

[8] X. H. Zhang, W. S. Lin and P. Xue, "Improved estimation for just-noticeable visual distortion”, Signal Processing, vol. 85, (2005).

[9] O. Guleryuz, "Weighted overcomplete denoising", The Asilomar Conference on Signals, Systems and Computers, (2003) November 9-12, Pacic Grove, CA, USA.

[10] K. Dabov, A. Foi, V. Katkovnik and K. Egiazarian, "A nonlocal and shape-adaptive transform-domain collaborative filtering", International workshop Local and Non-local Approximation in Image Processing, (2008) August 23-24, Lausanne, Switzerland.

[11] K. Dabov, A. Foi, V. Katkovnik and K. Egiazarian, "BM3D image denoising with shape-adaptive principal component analysis", Proceedings of workshop on signal processing with adaptive sparse structured representations, (2009) April 6-9, Saint-Malo, France. 


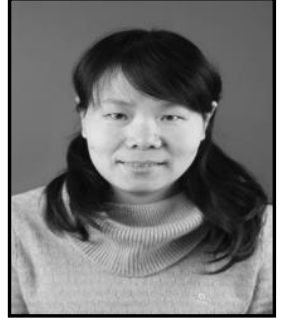

Xuemei Wang, She received her B.S. degree from Changchun College of Posts and Telecommunications, China in 2000 and M.S. degree from Nanjing University of Posts and Telecommunications, China in 2003. Now, she is working toward the Ph.D. degree in Nanjing University of Posts and Telecommunications, China. Her research interests mainly include computer vision, computer networks.

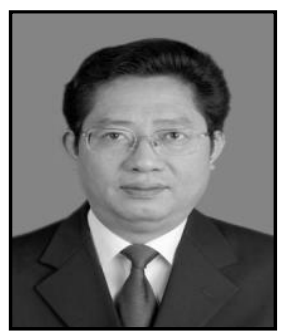

Dengyin Zhang, $\mathrm{He}$ received the B.S. degree in telecommunication engineering, the M.Sc. degree in Circuit, Signal and System, and the $\mathrm{PhD}$ degree in Signal and Information Processing from Nanjing University of Posts and Telecommunications, Nanjing, China, in 1986, 1989, and 2004, respectively.. He was elected as a master tutor and doctoral tutor in 2000 and 2010 respectively. His main research interest is in the field of signal and information processing, networking technique, Voice over IP (VoIP), and information security.

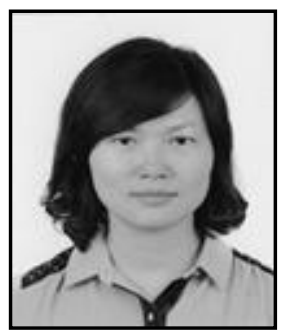

Min Zhu, She received her B.S. degree from Nanjing University of Posts and Telecommunications, China in 2002 and M.S. degree from Beijing University of Posts and Telecommunications, China in 2005. Now, she is working toward the Ph.D. degree in Nanjing University of Posts and Telecommunications, China. Her research interests mainly include routing protocol and algorithm design.

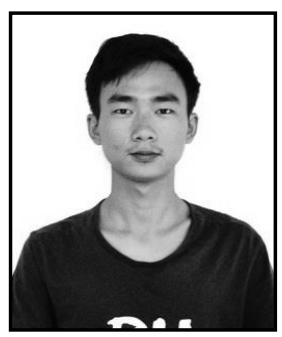

Yingtian Ji, He obtained his B.S. degree in the Communication Engineering from Nanjing University of Posts and Telecommunications, China in 2013. Now, he is working towards the M.S. degree in the Communication and Information Institute. His current research interests are in compressed sensing and distributed video coding.

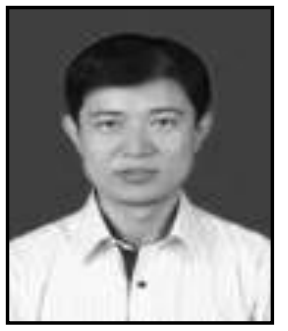

Jin Wang He received his B.S. and M.S. degree from Nanjing University of Posts and Telecommunications, China in 2002 and 2005, respectively. He received Ph.D. degree from Kyung Hee University Korea in 2010. Now, he is a professor in the College of Information Engineering, Yangzhou University. His research interests mainly include routing protocol and algorithm design, network performance evaluation and optimization for wireless ad hoc and sensor networks. He is a member of the IEEE and ACM. 
International Journal of Signal Processing, Image Processing and Pattern Recognition Vol. 8, No. 4 (2015) 